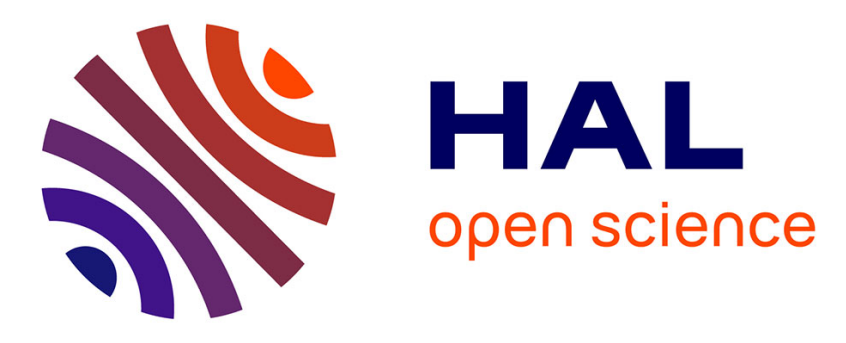

\title{
Embodied Evolution of Self-Organised Aggregation by Cultural Propagation
}

\author{
Nicolas Cambier, Vincent Frémont, Vito Trianni, Eliseo Ferrante
}

\section{To cite this version:}

Nicolas Cambier, Vincent Frémont, Vito Trianni, Eliseo Ferrante. Embodied Evolution of SelfOrganised Aggregation by Cultural Propagation. 11th International Conference on Swarm Intelligence (ANTS 2018), Oct 2018, Rome, Italy. pp.351-359, 10.1007/978-3-030-00533-7_29 . hal-01867727

\section{HAL Id: hal-01867727 https://hal.science/hal-01867727}

Submitted on 4 Sep 2018

HAL is a multi-disciplinary open access archive for the deposit and dissemination of scientific research documents, whether they are published or not. The documents may come from teaching and research institutions in France or abroad, or from public or private research centers.
L'archive ouverte pluridisciplinaire HAL, est destinée au dépôt et à la diffusion de documents scientifiques de niveau recherche, publiés ou non, émanant des établissements d'enseignement et de recherche français ou étrangers, des laboratoires publics ou privés. 


\title{
Embodied Evolution of Self-Organised Aggregation by Cultural Propagation
}

\author{
Nicolas Cambier ${ }^{1}$, Vincent Frémont ${ }^{1}$, Vito Trianni ${ }^{2}$ and Eliseo Ferrante ${ }^{3}$ \\ 1 Sorbonne universités, Université de technologie de Compiègne, \\ UMR CNRS 7253 Heudiasyc, Compiègne, France \\ nicolas.cambier@hds.utc.fr, vincent.fremont@hds.utc.fr \\ 2 Institute of Cognitive Sciences and Technologies, National Research Council, \\ Rome, Italy vito.trianni@istc.cnr.it \\ 3 Laboratory of Socio-ecology and Social Evolution, KU Leuven, Leuven, Belgium \\ eliseo.ferrante@kuleuven. be
}

\begin{abstract}
Probabilistic aggregation is a self-organised behaviour studied in swarm robotics. It aims at gathering a population of robots in the same place, in order to favour the execution of other more complex collective behaviours or tasks. However, probabilistic aggregation is extremely sensitive to experimental conditions, and thus requires specific parameter tuning for different conditions such as population size or density. To tackle this challenge, in this paper, we present a novel embodied evolution approach for swarm robotics based on social dynamics. This idea hinges on the cultural evolution metaphor, which postulates that good ideas spread widely in a population. Thus, we propose that good parameter settings can spread following a social dynamics process. Testing this idea on probabilistic aggregation and using the minimal naming game to emulate social dynamics, we observe a significant improvement in the scalability of the aggregation process.
\end{abstract}

\section{Introduction}

Aggregation processes are extremely common in Nature [10], and consist in animals aggregating in common areas in the environment. They have been studied in a variety of biological systems $[14,20]$ and also implemented on distributed robotic systems $[18,17,19]$, according to the swarm robotics ethos of getting inspiration from natural phenomena to solve engineering problems [25], while relying only on local interactions [7]. Furthermore, aggregation is a prerequisite for other cooperative behaviours [16].

There are many approaches to designing self-organised aggregation, from evolutionary solutions [31] to minimal deterministic models [19]. Probabilistic approaches are most commonly used [3] because of their simple implementation and direct natural inspiration $[20,18,5]$. Nevertheless, the aggregation quality obtained using probabilistic approaches is extremely sensitive to experimental conditions such as population size [3] or agent's capabilities (e.g., speed, communication range) [13], and therefore requires supervised tuning of internal model 
parameters in order to be effective in a specific setting [27,3]. Obviously, this necessity to tune collective behaviours to specific environmental conditions hinders both scalability and flexibility, both desired features of swarm robotics [7].

Evolutionary swarm robotics is the approach typically used for synthesising collective behaviours for specific experimental settings via off-line parameter tuning $[21,32,31]$. An approach of on-line parameter tuning is instead represented by embodied evolution [8], whereby parameters controlling the robot behaviour are continuously adapted as a result of the interaction among robots and between robots and environment. Here, the evolutionary process is not driven by an explicit fitness measure that evaluates the quality of the collective behaviour, but emerges implicitly from the dynamics of interaction among the agents [4]. This approach with implicit fitness characterises algorithms like Environment-driven Distributed Evolutionary Adaptation (EDEA) [9] and subsequent extensions such as MONEE [22]. These algorithms are inspired by natural evolution, and require (i) a high mobility of the agents to spread their genomes, and (ii) a survival criteria to decide whether individuals live (and reproduce) or die, usually implemented by means of a foraging metaphor. Both these criteria are hard to meet in self-organised aggregation, as agents aggregated on different clusters hardly communicate between each other, and a new survival criteria would thus need to be devised. Hence, different evolutionary dynamics must be devised.

In this paper, we propose a novel embodied evolutionary process inspired by the cultural evolution metaphor [29] that postulates that good ideas spread widely in a population as a result of social dynamics [12]. In our model, social dynamics are coupled with self-organised aggregation dynamics: parameters beneficial for aggregation spread widely in the robot swarm.

As a model of cultural evolution, we use the Naming Game (NG), which was developed to study the evolution of human language through statistical physics [2] and artificial life experiments [28]. The NG has actually already been studied within robotic swarms $[30,11]$, whereby the swarm dynamics (e.g., random walk and aggregation) and the NG had a mutual effect on each other. However, to the best of our knowledge, there has been no attempt to use the NG as an embodied evolution approach. In this paper, we move towards this direction by studying the dynamics of self-organised aggregation when coupled with the naming game framed as a cultural evolutionary process. The model is introduced in Section 2, while, in Section 3, we present and discuss experimental observations. Section 4 concludes the paper with an outlook of future research.

\section{Model}

The baseline aggregation controller we used is described in Section 2.1, while in Section 2.2, we describe the NG protocol and how it can be coupled with aggregation in our cultural evolution framework. 


\subsection{Self-organised Aggregation Controller}

One of the first models of probabilistic aggregation in swarm robotics takes inspiration from cockroaches collective behaviour model $[14,20,18]$. Here, agents move within an arena following a random walk [15]. They decide to stop according to some probability dependent on the number $n$ of perceived neighbours,as observed in cockroaches [20]. To prevent the creation of a large number of small clusters, exploration is introduced by allowing agents to leave their cluster with a probability inversely proportional to $n$.

As in previous models [13], we implemented a PFSA (see Figure 1b) with two states: WALK (random walk as in [23]) and STAY. To gain a higher control over the aggregation process, we replaced the transitions probabilities in [13] (Figure 1a) with probability functions with parametrisable steepness, allowing to strengthen or weaken the agents' alignment and dispersion at will (see Figure 1c). For both $P_{\text {Join }}$ and $P_{\text {Leave }}$, we used exponential decay functions which, for our baseline, were tuned to fit the values in Figure 1a:

$$
P_{\text {Join }}=0.03+0.48 *\left(1-e^{-a n}\right)
$$

In this equation, $a$ is a parameter that handles the strength of the alignment. Indeed $P_{\text {Join }}$ becomes steeper as $a$ increases.

$P_{\text {Leave }}$, on the other hand, is a straightforward exponential decay function:

$$
P_{\text {Leave }}=e^{-b n}
$$

Here, $b$ handles the strength of the dispersion as $P_{\text {Leave }}$ becomes steeper when $b$ increases, and thus dispersion weakens. Figure 1c shows the fitted values of these functions to the baseline values from Figure 1a.

We can understand the effect of parameters $a$ and $b$ as strengthening (increase $a$ or $b$ ) or weakening (decrease $a$ or $b$ ) the cohesion of a cluster. A trade-off between these two forces must be found: If cohesion is too weak (low $a$ and $b$ ), no durable cluster will form; if cohesion is too strong (high $a$ and $b$ ), the robots aggregate in several sparse and static clusters that never break. Following the above observations, we formulate the following premise:

Premise 1 Robots with (near) optimal parameters $a$ and $b$ have more neighbours, on average, than robots with suboptimal parameters.

Based on this premise we build an implicit fitness for embodied evolution.

\subsection{Cultural Evolution}

Self-organised aggregation and the minimal naming game (MNG) $[2,1]$ have been coupled as in [11]. Each agent possesses an individual lexicon (i.e., a list of words) and can be both a speaker (only when in the STAY state) and hearer (at all times). A speaker communicates a word from its lexicon to its neighbours, or generates a new one when the lexicon is empty. The neighbours in the speaker's communication range (i.e. the hearers) receive this word. If the word is already 


\begin{tabular}{|c|ll|}
\hline $\mathrm{n}$ & $P_{\text {Join }}$ & $P_{\text {Leave }}$ \\
\hline 0 & 0.03 & n.a. \\
1 & 0.42 & $1 / 49$ \\
2 & 0.50 & $1 / 424$ \\
3 & 0.51 & $1 / 700$ \\
$4+$ & 0.51 & $1 / 1306$ \\
\hline
\end{tabular}

(a)

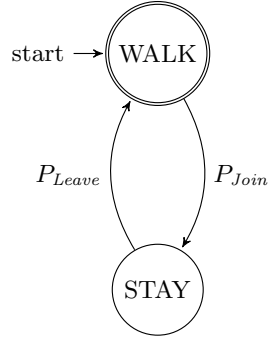

(b)

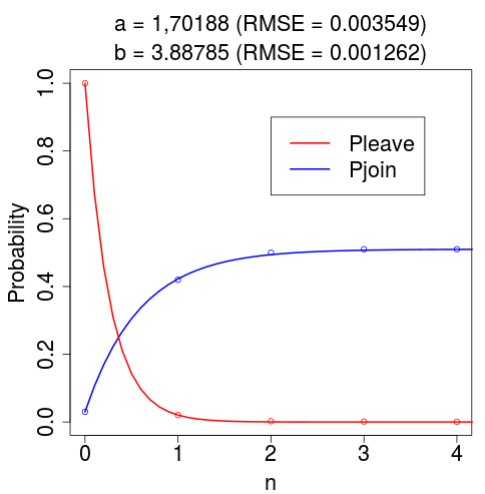

(c)

Fig. 1. (a) Averaged probability to join and leave a cluster as computed by [13] from observation of gregarious arthropods [20]. (b) PFSA of our aggregation controller. (c) Plotting of $P_{\text {join }}$ and $P_{\text {Leave }}$ according to (resp.) Eq. 1 and 2 and parametrised with the values for $a$ and $b$ that best fit the values from Table 1a [13] (circles).

known to the hearer, the game is a success and the hearer deletes all words from its lexicon except for the one it just received. Otherwise, the game is a fail and the hearer adds the word to its lexicon. In our implementation, the amount of a hearer's successful games in a time-step is used as $n$ for the computation of the transition probabilities in the PFSA.

Moreover, the MNG presents one interesting feature: words promoted by agents with more neighbours have a higher chance to eventually become the chosen name for the predetermined topic, at least in static networks [1]. Therefore, we formulate a second premise:

Premise 2 Words promoted by robots with more neighbours spread more on average.

From Premises 1 and 2, we conclude that words promoted by robots with (near) optimal parameters $a$ and $b$ should propagate more on average. Therefore, by using an encoding of the values of $a$ and $b$ as the words used in the MNG, we close a positive feedback loop whereby better parameters propagate more and, as they are shared by new robots, propagate even more.

In our experiments, we used the concatenation of the encoded values of $a$ and $b$ as the "meme" of our cultural evolution process. We assumed a minimal message size of one byte, and each parameter was therefore expressed with four bits. With these four bits we coded the $[1.25,5]$ interval with steps of 0.25 .

The above described MNG can converge only to a word generated by speakers at the beginning of an experiments. To allow for novelty, we added noise to the messages following the noisy channel model [26]. Noise has two effects in our implementations. Firstly, it acts as mutation operator and allows to explore the solution landscape [33]. Secondly, it impacts the quantity of (un)successful games 
as it can mutate a word known to the hearer to an unknown one, thus slowing down convergence time of the MNG and making it more compatible with the time-scale of self-organised aggregation. However, a high mutation rate $m$ may completely prevent the MNG from converging.

\section{Experimental Results}

In the experiments, we used MarXbots [6] simulated within the ARGoS simulator [24]. They moved at a speed of $10 \mathrm{~cm} / \mathrm{s}$ and with a communication range of $70 \mathrm{~cm}$ within a circular arena of constant radius $r=10 \mathrm{~m}$. We studied three different population sizes $N=\{25,50,100\}$ and evaluated the aggregation behaviour using the cluster metric in [19], which is the ratio between the size of the biggest cluster and the swarm size $N$.

To highlight the dynamics produced by embodied evolution, we contrasted it with selected non-evolving instances, namely the baseline controller obtained by fitting the parameters $a$ and $b$ to the probability table from [13], and the $o p$ timal controller obtained with the parameter settings that maximise the cluster metric separately for each swarm size through brute-force search. Therefore we performed:

- 20 runs of embodied evolution with mutation rate $m=0.001$

- 20 runs of the baseline controller featuring fixed parameters $(a, b)=$ $(1.70188,3.88785)$, set to fit [13]

- 10 runs of the optimal controller with fixed parameters:

- $N=25:(a, b)=(2.25,3.5)$

- $N=50:(a, b)=(1.25,2.0)$

- $N=100:(a, b)=(1.25,1.25)$

In addition to the cluster metric, we recorded the variation over time of the number of clusters formed and the number of free agents. The average figures are shown in Figure 2. It is possible to notice that the evolutionary model fails to produce stable aggregates when $N=25$. This is because the MNG is particularly slow at low densities, because interactions among agents happen with very low probability [30,11]. As a consequence, the number of successful games is small - also due to mutations disturbing the language dynamics - and clusters quickly disband. However, as $N$ increases, we can see that embodied evolution presents dynamics that are very close to the baseline aggregation behaviour [13], i.e. a short phase of building aggregates followed by stagnation. For $N=100$, embodied evolution attains values for the cluster metric that are larger than the baseline controller. Additionally, evolution has different dynamics from those of the baseline: Almost all agents with the baseline behaviour stay in clusters after the build-up phase, whilst the evolutionary model continues to explore for a longer time and never entirely stops. This is partly the consequence of the MNG and its mutation-induced failures (see Section 2.2), but also demonstrates a better handling of the cohesion trade-off (see Section 2.1), which explains our model's higher scalability, especially for large $N$. The optimal controllers, 

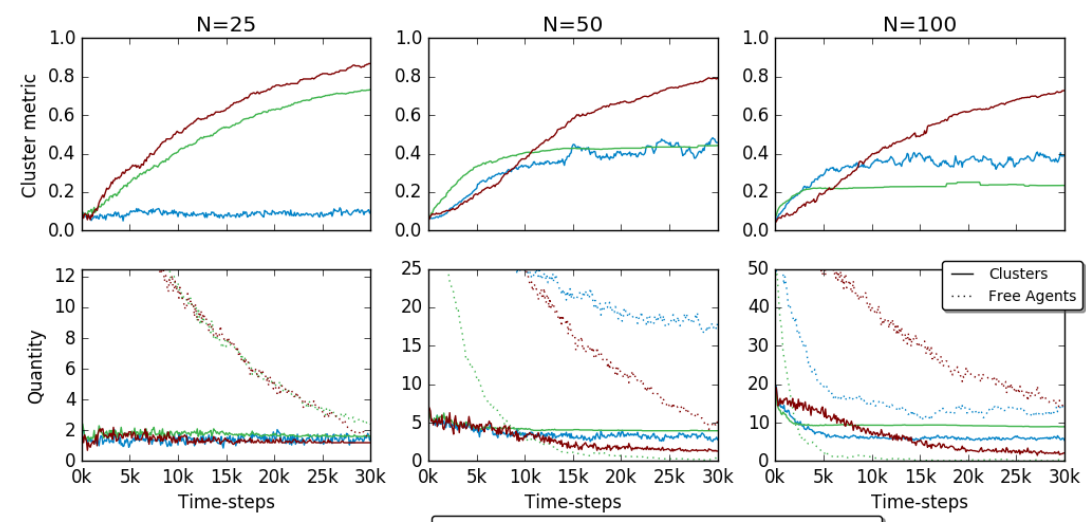

$$
\text { - Evolving }- \text { Baseline }- \text { Optimal }
$$

Fig. 2. Averaged time evolution over $i$ runs of the three variations: embodied evolution $(i=20)$, baseline controller with parameters fixed to match the probability table from [13], $i=20)$ and optimal $(i=10)$.

instead, slowly and constantly build up a large aggregate, maintaining at the same time a large fraction of exploring robots. This slow process represents the only means to increase the size of the largest cluster at the expenses of small clusters, when parameters are fixed and the system is homogeneous. However, a very specific parameterisation is necessary to observe this behaviour, especially for large $N$.

We speculate that the efficiency of the MNG (and, thus, of embodied evolution) depends on the amount of interactions and, thus, can not work in low density settings. To test this, we confront three experimental conditions in which density of robots is maintained constant: $N=25$ with $r=5 m, N=50$ with $r=7 m$, and $N=100$ with $r=10 \mathrm{~m}$. We contrast embodied evolution with the baseline controller and with the optimal controller obtained with brute force search for $N=25$ and $r=5 \mathrm{~m}$. The latter is tested also in the other conditions, to assess whether fixed parameterisation optimal on a given setting also perform well in other settings. The results are presented in Figure 3. We can see that, with sufficient robot density, the evolutionary model initially performs as well as the baseline behaviour [13] and not too distant from the optimal behaviour. However, embodied evolution scales up better than either of the fixed-parameters alternatives. We conclude that embodied evolution represents a promising solution for scalable behaviour rather than optimal probabilistic aggregation, provided that the density of robots remains sufficiently high.

\section{Conclusions}

In this paper, we presented a novel embodied evolution approach for swarm robotics based on social dynamics. The main underlying idea is coupling opinion 

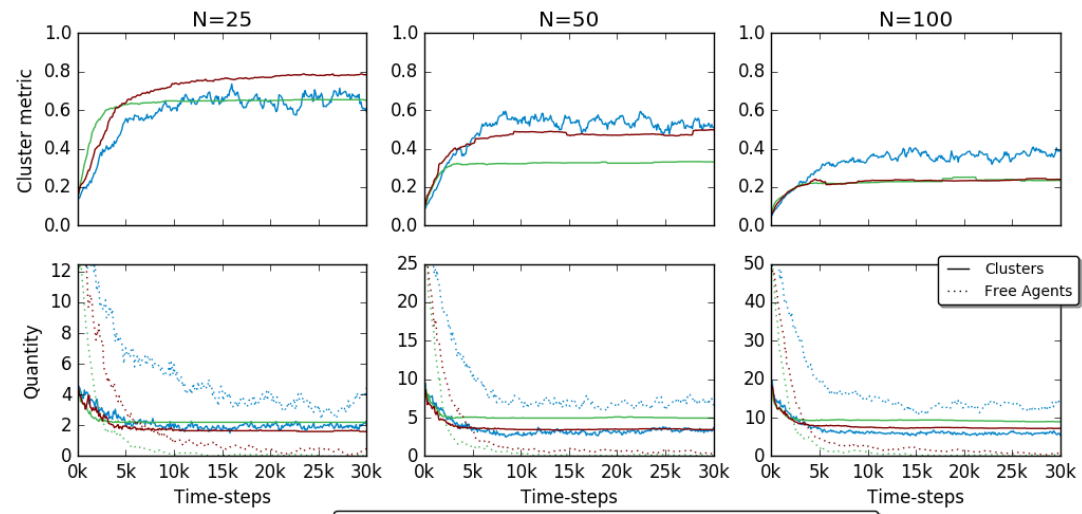

Evolving Optimal(for $\mathrm{N}=25)$

Fig. 3. Averaged time evolution over 20 runs of the three variations with constant density: embodied evolution, baseline behaviour and optimal controller for $N=25$ and $r=5 m$ (i.e. $(a, b)=(1.5,2.75))$.

spreading in the population with the self-organising aggregation process. Inspired by the cultural evolution metaphor, which postulates that good ideas spread widely in a population, we propose that good parameters of a self-organising behaviour can spread following a social dynamics process, leading to a swarm capable of adapting its behaviour to the current environmental conditions. This is possible to the extent that these coupled dynamics and the opinion spreading are self-sustaining. To test this idea we considered probabilistic aggregation as the self-organising swarm behaviour and the minimal naming game as a model of social dynamics. Experimental results show that the proposed embodied evolution process autonomously selects performing parameters at different scales provided that an adequate robot density is present.

Our future work will be to (i) evaluate our model's flexibility and adaptivity by varying the size $\mathrm{N}$ of the population as well as the environment's features within runs, (ii) study the criticality of the mutation rate for our algorithm's performances and (iii) assessing its efficiency as a natural communication noise in an embodied setting.

Acknowledgments. This work was funded in the framework of the Labex MS2T. It was supported by the French Government, through the program "Investments for the future" managed by the National Agency for Research (Reference ANR-11-IDEX-0004-02). This work was also carried out and funded by the European Regional Development Fund (ERDF) and by the Regional Council of Hauts de France. Vito Trianni acknowledges support from the project DICE (FP7 Marie Curie Career Integration Grant, ID: 631297). 


\section{References}

1. Baronchelli, A.: Role of feedback and broadcasting in the naming game. Physical Review E 83(4), 046103 (2011)

2. Baronchelli, A., Felici, M., Loreto, V., Caglioti, E., Steels, L.: Sharp transition towards shared vocabularies in multi-agent systems. Journal of Statistical Mechanics: Theory and Experiment 2006(06), P06014 (2006)

3. Bayindir, L., Sahin, E.: Modeling self-organized aggregation in swarm robotic systems. In: Swarm Intelligence Symposium, 2009. SIS'09. IEEE. pp. 88-95. IEEE (2009)

4. Bianco, R., Nolfi, S.: Toward open-ended evolutionary robotics: evolving elementary robotic units able to self-assemble and self-reproduce. Connection Science 16(4), 227-248 (2004)

5. Bodi, M., Thenius, R., Szopek, M., Schmickl, T., Crailsheim, K.: Interaction of robot swarms using the honeybee-inspired control algorithm beeclust. Mathematical and Computer Modelling of Dynamical Systems 18(1), 87-100 (2012)

6. Bonani, M., Longchamp, V., Magnenat, S., Rétornaz, P., Burnier, D., Roulet, G., Vaussard, F., Bleuler, H., Mondada, F.: The marxbot, a miniature mobile robot opening new perspectives for the collective-robotic research. In: Intelligent Robots and Systems (IROS), 2010 IEEE/RSJ International Conference on. pp. 4187-4193. $\operatorname{IEEE~(2010)~}$

7. Brambilla, M., Ferrante, E., Birattari, M., Dorigo, M.: Swarm robotics: a review from the swarm engineering perspective. Swarm Intelligence 7(1), 1-41 (2013)

8. Bredeche, N., Haasdijk, E., Prieto, A.: Embodied evolution in collective robotics: A review. Frontiers in Robotics and AI 5, 12 (2018)

9. Bredeche, N., Montanier, J.M.: Environment-driven embodied evolution in a population of autonomous agents. In: International Conference on Parallel Problem Solving from Nature. pp. 290-299. Springer (2010)

10. Camazine, S.: Self-organization in biological systems. Princeton University Press (2003)

11. Cambier, N., Frémont, V., Ferrante, E.: Group-size regulation in self-organised aggregation through the naming game. In: International Symposium on Swarm Behavior and Bio-Inspired Robotics (SWARM 2017). Kyoto, Japan (Oct 2017), https://hal .archives-ouvertes.fr/hal-01679600

12. Castellano, C., Fortunato, S., Loreto, V.: Statistical physics of social dynamics. Reviews of Modern Physics 81(2), 591-646 (2009)

13. Correll, N., Martinoli, A.: Modeling and designing self-organized aggregation in a swarm of miniature robots. The International Journal of Robotics Research 30(5), 615-626 (2011)

14. Deneubourg, J.L., Lioni, A., Detrain, C.: Dynamics of aggregation and emergence of cooperation. The Biological Bulletin 202(3), 262-267 (2002)

15. Dimidov, C., Oriolo, G., Trianni, V.: Random Walks in Swarm Robotics: An Experiment with Kilobots. In: Dorigo, M., Birattari, M., Li, X., López-Ibáñez, M., Ohkura, K., Pinciroli, C., Stützle, T. (eds.) Swarm Intelligence: 10th International Conference, ANTS 2016, Brussels, Belgium, September 7-9, 2016, Proceedings, pp. 185-196. Springer International Publishing (2016)

16. Dorigo, M., Trianni, V., Şahin, E., Groß, R., Labella, T.H., Baldassarre, G., Nolfi, S., Deneubourg, J.L., Mondada, F., Floreano, D., et al.: Evolving self-organizing behaviors for a swarm-bot. Autonomous Robots 17(2), 223-245 (2004) 
17. Garnier, S., Jost, C., Gautrais, J., Asadpour, M., Caprari, G., Jeanson, R., Grimal, A., Theraulaz, G.: The embodiment of cockroach aggregation behavior in a group of micro-robots. Artificial life 14(4), 387-408 (2008)

18. Garnier, S., Jost, C., Jeanson, R., Gautrais, J., Asadpour, M., Caprari, G., Theraulaz, G.: Aggregation behaviour as a source of collective decision in a group of cockroach-like-robots. In: European Conference on Artificial Life. pp. 169-178. Springer (2005)

19. Gauci, M., Chen, J., Li, W., Dodd, T.J., Groß, R.: Self-organized aggregation without computation. The International Journal of Robotics Research 33(8), 1145$1161(2014)$

20. Jeanson, R., Rivault, C., Deneubourg, J.L., Blanco, S., Fournier, R., Jost, C., Theraulaz, G.: Self-organized aggregation in cockroaches. Animal behaviour 69(1), 169-180 (2005)

21. Nolfi, S., Floreano, D.: Evolutionary robotics: The biology, intelligence, and technology of self-organizing machines. MIT press (2000)

22. Noskov, N., Haasdijk, E., Weel, B., Eiben, A.E.: Monee: Using parental investment to combine open-ended and task-driven evolution. In: European Conference on the Applications of Evolutionary Computation. pp. 569-578. Springer (2013)

23. Nouyan, S., Dorigo, M.: Chain based path formation in swarms of robots. In: International Workshop on Ant Colony Optimization and Swarm Intelligence. pp. 120-131. Springer (2006)

24. Pinciroli, C., Trianni, V., O’Grady, R., Pini, G., Brutschy, A., Brambilla, M., Mathews, N., Ferrante, E., Di Caro, G., Ducatelle, F., et al.: Argos: a modular, parallel, multi-engine simulator for multi-robot systems. Swarm intelligence 6(4), 271-295 (2012)

25. Şahin, E.: Swarm robotics: From sources of inspiration to domains of application. In: International workshop on swarm robotics. pp. 10-20. Springer (2004)

26. Shannon, C.: (1948)," a mathematical theory of communication", bell system technical journal, vol. 27, pp. 379-423 \& 623-656, july \& october (1948)

27. Soysal, O., Sahin, E.: Probabilistic aggregation strategies in swarm robotic systems. In: Swarm Intelligence Symposium, 2005. SIS 2005. Proceedings 2005 IEEE. pp. 325-332. IEEE (2005)

28. Steels, L.: A self-organizing spatial vocabulary. Artificial Life 2(3), 319-332 (1995)

29. Steels, L.: Modeling the cultural evolution of language. Physics of Life Reviews 8(4), 339-356 (2011)

30. Trianni, V., De Simone, D., Reina, A., Baronchelli, A.: Emergence of consensus in a multi-robot network: from abstract models to empirical validation. IEEE Robotics and Automation Letters 1(1), 348-353 (2016)

31. Trianni, V., Groß, R., Labella, T.H., Şahin, E., Dorigo, M.: Evolving aggregation behaviors in a swarm of robots. In: European Conference on Artificial Life. pp. 865-874. Springer (2003)

32. Trianni, V., Nolfi, S., Dorigo, M.: Evolution, Self-organization and Swarm Robotics. In: Swarm Intelligence, pp. 163-191. Springer Berlin Heidelberg, Berlin, Heidelberg (2008)

33. Winfield, A.F., Erbas, M.D.: On embodied memetic evolution and the emergence of behavioural traditions in robots. Memetic Computing 3(4), 261-270 (2011) 